\title{
SPATIAL STATISTICS OF TEXTONS
}

\author{
Gary Dahme Eraldo Ribeiro \\ Department of Computer Sciences \\ Florida Institute of Technology \\ Melbourne, FL, USA \\ Email: gdahme@fit.edu, eribeiro@cs.fit.edu \\ Department of Biological Sciences \\ Florida Institute of Technology \\ Melbourne, FL, USA \\ Email: mbush@fit.edu
}

Keywords: Texture classification, pollen, spatial statistics.

\begin{abstract}
Texture classification is one of the most studied and challenging problems in computer vision. A key requirement of successful texture classification algorithms is their ability to quantify the complex nature and diversity of real world textures. Recent developments in automatic texture classification have demonstrated the effectiveness of modeling texture elements as cluster centers of responses of a filter bank. Such methods rely primarily on similarity measurements of frequency histograms of vector quantized versions of the target texture. A main problem with these approaches is that pure frequency histograms fail to explicitly account for important spatial interaction between learned texture elements. Spatial interaction is key to classification when analyzing textures that have similar texture element frequency but differ in the way the texture elements are distributed across the image. In this paper, we propose the use of co-occurrence statistics to account for the spatial interaction among learned texture elements. This is accomplished by calculating spatial co-occurrence statistics on the maps of the learned texture elements. We demonstrate the effectiveness of our method on images from the Brodatz album as well as natural textures from a tropical pollen database. We also present a comparison with a state-of-the-art method for texture classication. Finally, our experiments show that the use of spatial statistics help improve the classification rates for certain textures that present sparse and statistically non-stationary texture elements such as pollen grain textures.
\end{abstract}

\section{INTRODUCTION}

Recent developments in texture classification have demonstrated the effectiveness of representing texture elements as cluster centers of convolution responses of a filter bank. The main idea in these approaches is to create statistically representative appearance models that describe the repetitive patterns of a texture (Leung and Malik, 2001; Varma and Zisserman, 2004). These appearance models of texture elements are called textons. The modelling procedure consists of learning a dictionary of textons containing models of all representative texture elements in the training dataset. This is followed the construction of a vector quantized map of the texture image based on the texton dictionary. The vector quantized map is called "texton map" and comparisons between two textures is accomplished via a $\chi^{2}$ test of frequency histograms of their corresponding texton maps.

However, important spatial relationship between textons is lost during the process of histogram generation. As a result, chi-square comparison of texton his-

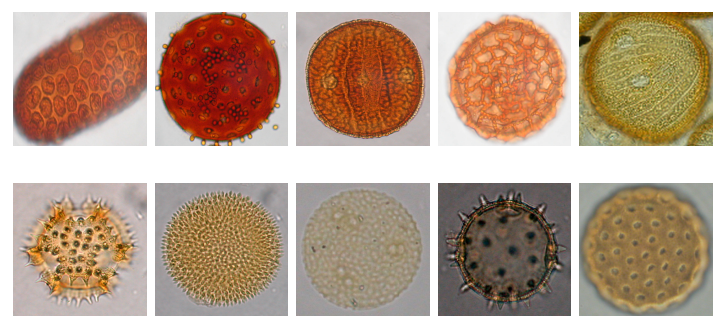

Figure 1: Optical microscopy images of pollen grains from the Neotropical Pollen Database (Mark and Wengs, 2006). Pollen grain textures usually present well defined and distinctive texture elements. Texture patterns spatial distribution tend to be sparse and quasi-regular.

tograms alone will not provide enough distinguishing power to classify textures that differ mostly in their spatial structure while having similar frequency statistics. A simple and effective way to solve this problem is by calculating spatial statistics on the texton maps and use these statistics for classification. 
In this paper, we propose the use of co-occurrence spatial statistics calculated on the texton maps as a means to improve classification of textures that differ mostly in their spatial structure. In the implementation proposed in this paper, we use the gray-level cooccurrence matrix (Haralick et al., 1973) to account for the spatial structure of textons. These statistics help improve classification of textures, especially in those textures that otherwise cannot be correctly classified using histogram-based methods.

We are particularly interested in the classification of textures that present similar texton frequency but differ in the way their textons are spatially distributed in the image. Examples of such textures include manmade ones such as buildings and cloth pattens, and natural ones such as coral reefs and pollen grains. In this paper, we show how the spatial structure of textons can be used for the recognition of pollen in optical microscope images (Bush, 2000; Mark and Wengs, 2006). We are currently working on the development of an automatic image-based algorithm for pollen recognition. Figure 1 shows examples of pollen grain images acquired using an optical microscope.

The remainder of this paper is organized as follows. In Section 2, we provide a concise review of the current related literature. In Section 3, we summarize the original texton-based classification framework. In Section 4, we describe the details of our method. In Section 5, we demonstrate the effectiveness of our algorithm on two texture datasets. Finally, in Section 6, we present our conclusions and plans for future work.

\section{RELATED WORK}

The literature on texture classification is extensive. In general, approaches can be divided into two main groups: 2D methods and 3D methods. The first group of methods model texture in terms of the spatial variations of albedo on a planar surface. The second group of methods attempts to model the reflectance variations due to 3D factors such as surface relief, camera viewpoint, and illumination. Representative of 2D methods include works based on Markov Random Fields, which model the spatial statistical relationships of pixels (Chellapa et al., 1985; Cross and Jain, 1983), and descriptive models based on filter banks (Leung and Malik, 1999). Recent work on texture classification has focused on the problem of modeling 3D appearance of surface materials (Leung and Malik, 2001; Varma and Zisserman, 2004; Dong and Chantler, 2005).

Leung and Malik (Leung and Malik, 1999; Leung and Malik, 2001) have introduced a descriptive model capable of encoding essential local structures and attributes of natural textures. Their method begins by representing each pixel of a texture as the convolution response of a bank of multi-scale and multi-orientation filters. The filter responses at each pixel location are concatenated into vectors. A KMeans clustering method (Duda et al., 2001)is applied to each filter response vector and the estimated cluster centers are chosen to be statistical representations of the texture elements or "textons". The idea of textons as cluster centers of filter responses has inspired several extensions of Leung and Malik's original method (Varma and Zisserman, 2004; Dong and Chantler, 2005; Cula and Dana, 2004; Zhu et al., 2005).

The strength of Leung and Malik's method is the ability to learn a statistically descriptive model of textures based on the responses of the filter bank. However, the method in its original version relies on similarity measurements between one-dimensional frequency histograms of vector quantized texture images. As a result, important information is lost in the process of histogram generation.

A possible solution to account for spatial structure is to represent the relationship among textons using spatial statistics measurements such as Markov Random Fields (Chellapa et al., 1985; Cross and Jain, 1983; Zhang et al., 2000) as well as descriptors of a co-occurence matrix (Haralick et al., 1973). In this paper, we propose the use of the well-known graylevel co-occurrence matrix as a way to capture spatial interaction among textons (Haralick et al., 1973). Next, we will show texton spatial interaction representation can help improve recognition rates for certain types of textures.

\section{TEXTONS AS CLUSTER CENTERS}

In this section, we briefly describe Leung and Malik's (Leung and Malik, 1999) approach to texture classification. The key idea is to model texture elements as cluster centers of convolution filter responses. Let $F$ be a bank of filters of several orientations and scales. Figure 2 shows an example of the filter bank used in (Leung and Malik, 1999; Leung and Malik, 2001). In the filter bank used in (Leung and Malik, 1999; Leung and Malik, 2001) there are 4 types of filters including an oriented filter set and an isotropic filter set. The oriented set is comprised of two groups, where each group has 6 different orientations and 3 different scales. The isotropic set consists of 12 filters each at different scales.

The process begins with the construction of a texton dictionary that will represent the information in each texture sample. Let $I(x, y)$ be a texture image. 


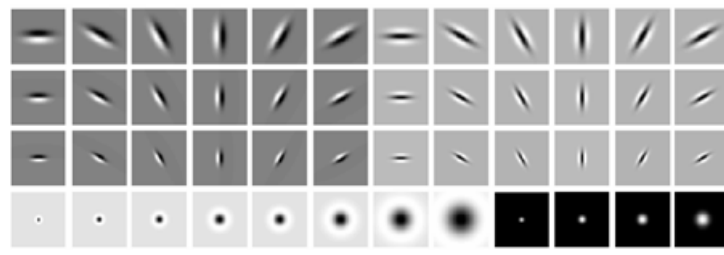

Figure 2: Filter bank consisting of 48 filters with varying orientations and scales (Leung and Malik, 2001). The oriented filters consists of two sets, each having 6 different orientations at 3 different scales. The remaining 12 are isotropic filters at different scales.

Image $I(x, y)$ is convolved with the filter bank $F$ to produce a set of filter responses as follows:

$$
R_{i}(x, y)=F_{i} * I(x, y), \quad i=1, \ldots, K .
$$

Where $\mathrm{K}$ is the total number of filters in the filter bank. In (Leung and Malik, 1999; Leung and Malik, 2001), $K$ is choosen to be 48 . By stacking the filter responses $R_{i}$, each pixel of $I(x, y)$ can now be represented by a $K$-dimensional feature vector $\mathbf{p}$ whose elements are the filter responses at each pixel location $(x, y)$. Each pixel in the texture image is represented by:

$$
\mathbf{p}(x, y)=\left[R_{1}(x, y), \ldots, R_{K}(x, y)\right]^{\top}
$$

The repetitive nature of textures implies that there will be many similar values of $\mathbf{p}$. To account for this redundancy, the vectors $\mathbf{p}$ are grouped using a K-Means clustering algorithm. The cluster centers produced by the K-means procedure are assumed to be statistical representations of the texture elements in the image and are called "textons". These textons are placed into set that represents a dictionary used to "explain" each texture in the training dataset. Describing a texture in terms of the texton dictionary is accomplished via a vector quantization procedure in which each pixel location of an image is labeled with the index corresponding to the closest texton in the dictionary. This creates an intermediate representation of the image that is called a texton map. Examples of texton maps generated from two distinct pollen grain textures are illustrated in the first column of Figure 3.

Finally, the classification stage is based on a chisquare similarity measure between frequency histograms of texton maps. The chi-square similarity is given by:

$$
\chi^{2}(\mathbf{p}, \mathbf{q})=\sum_{i} \frac{\left(p_{i}-q_{i}\right)^{2}}{p_{i}+q_{i}}
$$

where $p_{i}$ and $q_{i}$ are bins of two texton map histograms $\mathbf{p}$ and $\mathbf{q}$, respectively.

However, while the chi-square measure may be a suitable means for classification of textures exhibiting statistically stationary features (i.e., uniform level of regularity in spatial distribution), the case may arise where two textures that share similar frequency statistics nevertheless differ greatly in terms of spatial properties. In such an instance, a frequency histogram-based method would identify the two textures as belonging to the same class. On the other hand, an approach based on spatial statistics would be able to correctly distinguish between them. In Figure 3 , we show an example of this problem. As previously mentioned, the first column shows the texton maps of two very distinct pollen grain textures. The texture in the top row is composed by elongated texture patterns while the one in the bottom row has large blobs as texture elements. Yet, both textures produce almost identical texton frequency histograms using the method proposed by Leung and Malik. The second column (center) displays the Leung and Malik frequency histograms as generated from the corresponding texton maps.

\section{ADDING SPATIAL STATISTICS}

In this section, we describe how spatial statistics can help improve the classification of certain types of textures that cannot be correctly classified using texton frequency histogram-based methods.

A simple and effective way to represent spatial interaction among texture elements is by directly calculating spatial statistics on the texton maps. In our implementation, we propose the use of measurements based on the well-known gray-level cooccurrence matrix (GLCM) (Haralick et al., 1973). Co-occurrence matrices effectively capture spatial texture structure of an image. Given an image, the GLCM is a matrix in which each entry $(i, j)$ corresponds to the frequency of occurrences of the pair of intensity values $i$ and $j$ that are at a distance $d$ apart in the original image. A set of texture descriptors can be extracted from the co-occurrence matrix. In the original work by Haralick (Haralick et al., 1973), 16 texture descriptors were proposed. In our current implementation, we adopt the four mostly used descriptor. We calculate them at four angular intervals of 45 degrees. The descriptors we use are shown in Table 1.

Table 1: Co-occurrence Matrix Descriptors

\begin{tabular}{ll}
\hline \hline Contrast & $\sum_{i} \sum_{j}(i-j)^{2} P_{d}(i, j)$ \\
Correlation & $\sum_{i} \frac{\left(i-\mu_{i}\right)\left(j-\mu_{j}\right)}{\sigma_{\sigma} \sigma_{j}}$ \\
Energy & $\sum_{i} \sum_{j} P_{d}(i, j)^{2}$ \\
Homogeneity & $\sum_{i} \sum_{j} \frac{P_{d}(i, j)}{1+(i-j)^{2}}$ \\
\hline
\end{tabular}


In the equations listed in Table $1, P_{d}$ represents a gray-level co-occurrence matrix calculated at a distance interval $d$.

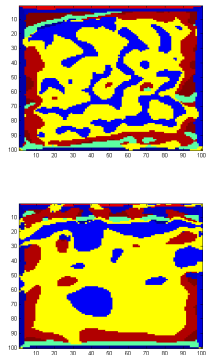

(a) Texton maps
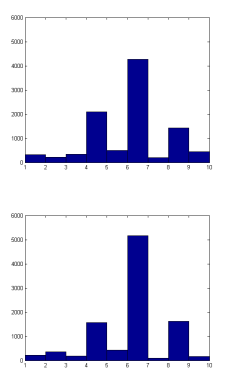

(b) Leung and Malik
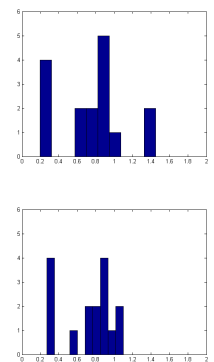

(c) Our Method
Figure 3: Two examples of similar histograms from different textures. Left column: texton maps for two distinct pollen grains. Center column: corresponding texton histograms. Right column: Histogram displaying values of GLCM features.

Figure 3 illustrates the importance of quantifying the spatial relationship between textons. The right column displays the values of our texture descriptor based of the co-occurrence matrix calculations on the corresponding texton maps in the first and second row of the first column, respectively. We display the values of our descriptor in histogram form for the purpose of illustration. The descriptors generated by our representation encodes the distinction between the textures. This is an example when the frequencybased method does not always provide enough information for the discrimination of certain textures.

\section{EXPERIMENTS}

The aim of our experiments was to demonstrate that the use of spatial statistics on the texton-based representation originally proposed by Leung and Malik (Leung and Malik, 2001) can help improve classification when textures differ mostly by the spatial structure of textons.

We begin by providing a comparison between our method and the histogram-based framework proposed by Leung and Malik (LM). Our experiments consist of two main comparisons. Firstly, we compare the classification results of the LM method with our method using 20 textures from the Brodatz album (Brodatz, 1966). The classification results are shown in Table 2 and Table 3, respectively. In the second set of experiments, we repeat the classification procedure using 10 images from our Neotropical Pollen Database (Mark and Wengs, 2006). The clas- sification results are shown in Table 4 and Table 5, respectively.

In Tables 2 through 5, columns represent known textures for which a database has been learned using the K-Means clustering as described in Section 3. The rows represent unknown textures that we wish to classify. Each cell in the tables represents the percentage of classification between a sample of novel textures and each texture in the learned training set. For illustration purposes, we display the texture images in each corresponding cell in the tables.

For classification results on the Brodatz textures, the overall recognition rate of our method was $88 \%$, whereas the LM approach was $96 \%$. Table 2 and Table 3 summarize the results of this classification experiment.

In the second part of the experiments, we apply our method to the problem of pollen recognition based on surface texture. The overall recognition rate of our method on pollen grain textures was $81 \%$, whereas the LM method was $69 \%$. Examples of the pollen grain subregions used are shown in Figure 4 . The source images from which the subregions were taken are shown in Figure 1 in the beginning of this paper. The results are presented in Table 4 and Table 5.
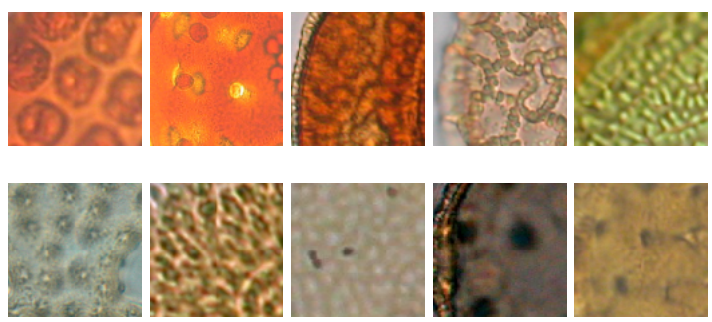

Figure 4: Subregions of pollen images (Mark and Wengs, 2006)

Pollen grain texture present two key differences when compared to textures in the Brodatz album and other available texture databases. Firstly, pollen texture do not present strong statistical regularity in the spatial arrangement of texture elements. Secondly, spatial arrangement of texture elements is the most distinctive feature for most pollen grains. The spatial arrangement is naturally captured by our descriptor and this allows it to achieve better recognition rates than the ML method for pollen grain textures.

Finally, in it current form, our method did not outperform the ML method for most textures in the Brodatz album. We believe that the main reason for this is the fact that Brodatz textures, in general, exhibit more statistically stationary behavior than our pollen textures. 
Table 2: Classification Percentage Matrix for Brodatz Textures (Texton Co-occurrence)

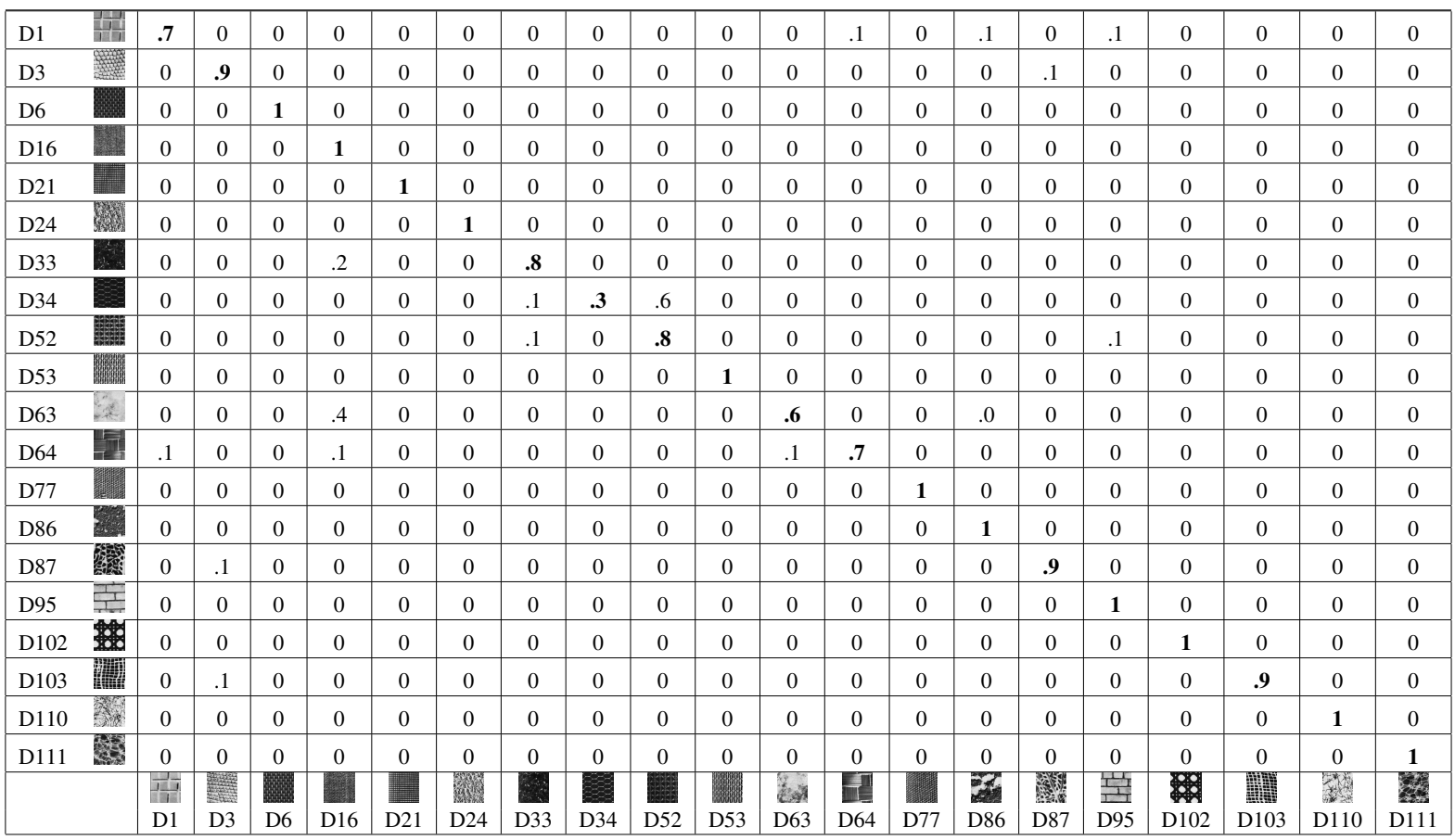

\section{CONCLUSIONS}

In this paper, we proposed the use of co-occurrence spatial statistics calculated on the texton maps as a means to improve classification of textures that differ mostly in their spatial structure.

We experimented our algorithm to set of texture images from the Brodatz album. We also apply the algorithm to the problem of recognizing pollen grain of a tropical pollen grain database. We are currently working on the development of an automated imagebase pollen recognition system. Our method achieved classification rates as high as $81 \%$ for the case of pollen grain textures. However, it did not significantly outperform current frequency histogram-based methods for most textures in the Brodatz album.

Frequency-based histograms are likely to produce high rates of classification for highly statistically stationary textures such as the ones in the Brodatz album and some of the available texture databases. However, methods relying on spatial statistics of texture elements are effective when applied non-regularly and sparsely distributed textures.

Our Future work include the further development of method presented in this paper by experimenting with larger number of co-occurrence descriptors. We also aim at investigating the use of Markov Random Fields representations to model texture element inter- action. For the pollen grain recognition system, we will investigate the inclusion of contour information to aid the recognition process.

\section{REFERENCES}

Brodatz, P. (1966). Textures: A Photographic Album for Artists and Designers. Dover, New York.

Bush, M. (2000). Deriving response matrices from central american modern pollen rain. Quaternary Research, 54:132-143.

Chellapa, R., Chatterjee, S., and Bagdazian, R. (1985). Texture synthesis and compression using gaussian-markov random fieldmodels. IEEE Trans. Systems, Man, and Cybernetics, 15:298303.

Cross, G. and Jain, A. (1983). Markov random field texture models. IEEE Trans. Pattern Analysis and Machine Intelligence, 5:25-39.

Cula, O. and Dana, K. J. (2004). 3d texture recognition using bidirectional feature histograms. International Journal of Computer Vision, 59:3360.

Dong, J. and Chantler, M. J. (2005). Capture and syn- 
Table 3: Classification Percentage Matrix for Brodatz Textures (Texton Frequency Histogram)

\begin{tabular}{|c|c|c|c|c|c|c|c|c|c|c|c|c|c|c|c|c|c|c|c|c|}
\hline 111 & .9 & 0 & 0 & 0 & 0 & 0 & 0 & 0 & 0 & 0 & 0 & 0 & 0 & 0 & 0 & 0 & 0 & 0 & .1 & 0 \\
\hline D3 & 0 & .9 & 0 & 0 & 0 & 0 & 0 & 0 & 0 & 0 & 0 & 0 & 0 & 0 & .1 & 0 & 0 & 0 & 0 & 0 \\
\hline D6 & 0 & 0 & 1 & 0 & 0 & 0 & 0 & 0 & 0 & 0 & 0 & 0 & 0 & 0 & 0 & 0 & 0 & 0 & 0 & 0 \\
\hline D16 & 0 & 0 & 0 & 1 & 0 & 0 & 0 & 0 & 0 & 0 & 0 & 0 & 0 & 0 & 0 & 0 & 0 & 0 & 0 & 0 \\
\hline D21 & 0 & 0 & 0 & 0 & 1 & 0 & 0 & 0 & 0 & 0 & 0 & 0 & 0 & 0 & 0 & 0 & 0 & 0 & 0 & 0 \\
\hline D24 & 0 & 0 & 0 & 0 & 0 & 1 & 0 & 0 & 0 & 0 & 0 & 0 & 0 & 0 & 0 & 0 & 0 & 0 & 0 & 0 \\
\hline D33 & 0 & 0 & 0 & 0 & 0 & 0 & 1 & 0 & 0 & 0 & 0 & 0 & 0 & 0 & 0 & 0 & 0 & 0 & 0 & 0 \\
\hline D34 & 0 & 0 & 0 & 0 & 0 & 0 & .1 & .9 & 0 & 0 & 0 & 0 & 0 & 0 & 0 & 0 & 0 & 0 & 0 & 0 \\
\hline D52 & 0 & 0 & 0 & 0 & 0 & 0 & 0 & 0 & 1 & 0 & 0 & 0 & 0 & 0 & 0 & 0 & 0 & 0 & 0 & 0 \\
\hline D53 & 0 & 0 & 0 & 0 & 0 & 0 & 0 & 0 & 0 & 1 & 0 & 0 & 0 & 0 & 0 & 0 & 0 & 0 & 0 & 0 \\
\hline D63 & 0 & 0 & 0 & 0 & 0 & 0 & 0 & 0 & 0 & 0 & 1 & 0 & 0 & .0 & 0 & 0 & 0 & 0 & 0 & 0 \\
\hline D64 & 0 & 0 & 0 & .1 & 0 & 0 & 0 & 0 & 0 & 0 & 0 & .9 & 0 & 0 & 0 & 0 & 0 & 0 & 0 & 0 \\
\hline D77 & 0 & 0 & 0 & 0 & 0 & 0 & 0 & 0 & 0 & 0 & 0 & 0 & 1 & 0 & 0 & 0 & 0 & 0 & 0 & 0 \\
\hline D86 & 0 & 0 & 0 & 0 & 0 & 0 & 0 & 0 & 0 & 0 & 0 & .2 & 0 & .8 & 0 & 0 & 0 & 0 & 0 & 0 \\
\hline D87 & 0 & .1 & 0 & 0 & 0 & 0 & 0 & 0 & 0 & 0 & 0 & 0 & 0 & 0 & .9 & 0 & 0 & 0 & 0 & 0 \\
\hline $\mathrm{D} 95$ 衰 & 0 & 0 & 0 & 0 & 0 & 0 & 0 & 0 & 0 & 0 & 0 & 0 & 0 & 0 & 0 & 1 & 0 & 0 & 0 & 0 \\
\hline $\mathrm{D} 102 \%$ & 0 & 0 & 0 & 0 & 0 & 0 & 0 & 0 & 0 & 0 & 0 & 0 & 0 & 0 & 0 & 0 & 1 & 0 & 0 & 0 \\
\hline D103 期飘 & 0 & 0 & 0 & 0 & 0 & 0 & 0 & 0 & 0 & 0 & 0 & 0 & 0 & 0 & 0 & 0 & 0 & 1 & 0 & 0 \\
\hline D110 & 0 & 0 & 0 & 0 & 0 & 0 & 0 & 0 & 0 & 0 & 0 & 0 & 0 & 0 & 0 & 0 & 0 & 0 & 1 & 0 \\
\hline D111 致 & 0 & 0 & 0 & 0 & 0 & 0 & 0 & 0 & 0 & 0 & 0 & 0 & 0 & 0 & 0 & 0 & 0 & 0 & 0 & .9 \\
\hline & $\begin{array}{l}\text { जiti } \\
\text { D1 }\end{array}$ & 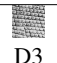 & D6 & D16 & D2 & D24 & 033 & & & & D6 & EI & & 3 & 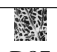 & 舆 & 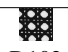 & 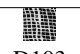 & N & $\begin{array}{r}378 \\
\text { D111 }\end{array}$ \\
\hline
\end{tabular}

thesis of 3d surface texture. International Journal of Computer Vision, 62:177-194.

Duda, R., P.E., H., and D.G., S. (2001). Pattern Classification. John Wiley and Sons, Inc.

Haralick, R. M., Shanmugam, K., and Dinstein, I. (1973). Textural features for image classification. IEEE Trans. on Systems, Man, and Cyberneticcs, SMC-3:610-621.

Leung, T. and Malik, J. (1999). Recognising surfaces using three-dimensional textons. In ICCV99, pages 1010-1017.

Leung, T. and Malik, J. (2001). Representing and recognizing the visual appearance of materials using three-dimensional textons. International Journal of Computer Vision, 43:29-44.

Mark, M. and Wengs, M. (2006). Introducing a new (freeware) tool for Palynology. Journal of Biogeography. (In Press).

Varma, M. and Zisserman, A. (2004). A statistical approach to texture classification from single images. International Journal of Computer Vision, 62:61-81

Zhang, J., Fieguth, P., and Wang, D. (2000). Random Field Models.

Zhu, S. C., en Guo, C., Wang, Y., and Xu, Z. (2005).
What are textons? International Journal of Computer Vision, 62(1-2):121-143. 
Table 4: Classification Percentage Matrix for Pollen Texture (Texton Co-occurrence)

\begin{tabular}{|c|c|c|c|c|c|c|c|c|c|c|}
\hline Jacobina macedoana & .9 & 0 & .1 & 0 & 0 & 0 & 0 & 0 & 0 & 0 \\
\hline Louteridium donnelsmithii & 0 & .6 & 0 & 0 & 0 & 0 & 0 & 0 & .4 & 0 \\
\hline Pachystachys lutea & .1 & .1 & .8 & 0 & 0 & 0 & 0 & 0 & 0 & 0 \\
\hline Ruellia graecizans & 0 & 0 & 0 & .4 & .3 & 0 & .3 & 0 & 0 & 0 \\
\hline Tricanthera gigantea & 0 & 0 & 0 & .2 & .8 & 0 & 0 & 0 & 0 & 0 \\
\hline Prenanthes alba & .3 & 0 & 0 & 0 & 0 & .7 & 0 & 0 & 0 & 0 \\
\hline Aleurites moluccana & 0 & 0 & 0 & 0 & 0 & 0 & 1 & 0 & 0 & 0 \\
\hline Bocconia frutesens & 0 & 0 & 0 & 0 & 0 & 0 & 0 & 1 & 0 & 0 \\
\hline Robinsonella mirandae & 0 & 0 & .1 & 0 & 0 & 0 & 0 & 0 & .9 & 0 \\
\hline Kochia scoparia & 0 & 0 & 0 & 0 & 0 & 0 & 0 & 0 & 0 & 1 \\
\hline & 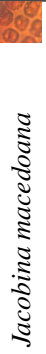 & 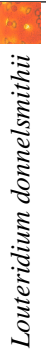 & 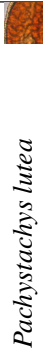 & 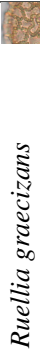 & 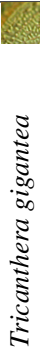 & 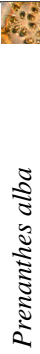 & 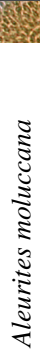 & 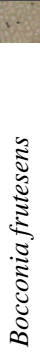 & 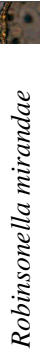 & 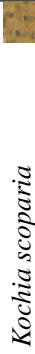 \\
\hline
\end{tabular}

Table 5: Classification Percentage Matrix for Pollen Textures (Texton Frequency Histogram)

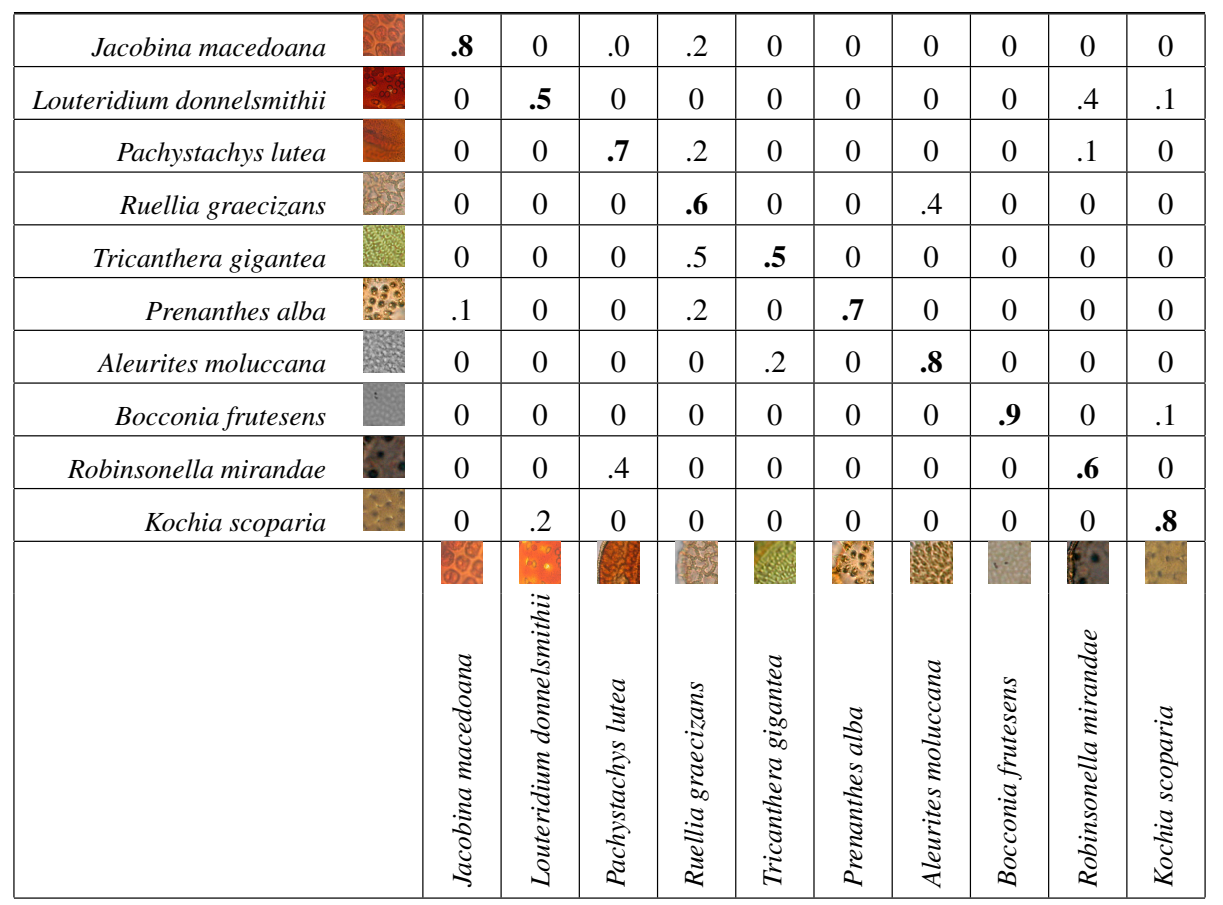

